\title{
2-Hydroxy-4-methoxybenzophenone Enhances the Suppression of Superoxide Anion Radicals Generated via UVA-induced Photosensitizing by t-Butyl Methoxydibenzoylmethane
}

\author{
Kazuhiro Yamaguchi* , Risa Ohtsuka, Koyuki Kaneko, Sonmine Che, Masahiro Maeda, \\ Hitoshi Masaki, and Tokuro Iwabuchi \\ School of Bioscience and Biotechnology, Tokyo University of Technology, 1404-1 Katakura-cho, Hachioji, Tokyo 192-0982, JAPAN
}

\begin{abstract}
Butyl-4'-methoxydibenzoylmethane (BMDM) is widely used throughout the world as a highly effective UVA absorber that can prevent the progression of photoaging in skin. However, due to its low photostability, BMDM is also known for the disadvantage of having a reduced capability to absorb UVA during prolonged exposure to sunlight. Although many studies have been carried out to overcome this disadvantage of BMDM, little attention has been paid to how the radicals generated from BMDM during UV exposure influence the skin. Therefore, the purpose of this study was twofold: One goal was to clarify the influence of radicals on human skin using cytotoxicity as a parameter. The second was to propose a solution that could reduce the radical formation while taking photostability into consideration. Using ESR spin trapping and superoxide dismutase (SOD) treatment, the radicals produced by the UV exposure of BMDM were shown to be superoxide anion radicals $\left(\cdot \mathrm{O}_{2}^{-}\right)$. HaCaT keratinocytes exposed to UVA in the presence of BMDM showed a significant reduction in cell viability, indicating that the radicals produced from BMDM have a harmful influence on the skin. UVA exposure coincidently led to a reduction of UVA absorbance by BMDM. Interestingly, 2-hydroxy-4-methoxybenzophenone (Benzophenone-3; BP3) reduced both the radical formation and the cytotoxicity resulting from the UVA-exposure of BMDM, while also restoring its UVA absorbance. In conclusion, the results show that BMDM and BP3 is an effective combination to reduce the influence of UVA-exposed BMDM on the skin and to prevent the loss of UVA absorbance by BMDM during UV exposure.
\end{abstract}

Key words: BMDM, 2-hydroxy-4-methoxybenzophenone, ESR, HaCaT, radicals, UVA absorber combination, photostability, superoxide dismutase, neutral red uptake assay

\section{Introduction}

It is widely recognized that ultraviolet radiation(UV) is a strong factor that initiates and promotes skin inflammation and accelerates the process of skin aging called photoaging $^{1,2)}$. Excessive exposure to UVB is easy to recognize as an erythemal skin response. However, since excessive UVA exposure does not cause a remarkably visible skin response, it is possible that we are unconsciously exposed to much higher doses of UVA than we imagine. Furthermore, since UVA is able to reach deeply into the dermis, it is considered that chronic UVA exposure to skin is a critical factor in the acceleration of skin aging as it causes alterations in the dermal matrix.
It has been well demonstrated that reactive oxygen species (ROS) are responsible for UVA-induced alterations of the dermal matrix. In general, UVA is recognized as a generator of ROS through photosensitizing reactions or the activation of NADPH oxidase. UVA generates singlet oxygen $\left({ }^{1} \mathrm{O}_{2}\right)$ through a photosensitizing reaction by exciting intracellular chromophores, such as flavoproteins ${ }^{3)}$. Furthermore, UVA stimulates the production of $\cdot \mathrm{O}_{2}{ }^{-}$ through the activation of NADPH oxidase due to increases in intracellular $\mathrm{Ca}^{2+}$ concentrations ${ }^{4)}$. ROS upregulates the mRNA expression levels of CCN1/Cyr61 (Cysteine-Rich Heparin-Binding Protein 61), which is a feedback protein for collagen synthesis, and of matrix metalloproteinase-1

\footnotetext{
*Correspondence to: Kazuhiro Yamaguchi, School of Bioscience and Biotechnology, Tokyo University of Technology, 1404-1 Katakura-cho, Hachioji, Tokyo 192-0982, JAPAN

E-mail: d1119006c9@edu.teu.ac.jp

Accepted April 21, 2020 (received for review December 26, 2019)

Journal of Oleo Science ISSN 1345-8957 print / ISSN 1347-3352 online

http://www.jstage.jst.go.jp/browse/jos/ http://mc.manusriptcentral.com/jjocs
} 
(MMP-1), which is an enzyme that degrades collagen, through EGF-R/JNK (epidermal growth factor receptor/ c-Jun N-terminal kinase) signaling. As a result, an imbalance between the synthesis and degradation of collagen occurs that results in the depletion of collagen in the dermis. Furthermore, ROS enhances the secretion of IL-8, which is a chemokine that recruits neutrophils, causing the degradation of elastic fibers in the papillary dermis by neutrophil elastase $\mathrm{e}^{5)}$. These facts indicate the importance of reducing intracellular ROS levels to prevent the progression of photoaging in the $\operatorname{skin}^{6-9)}$. Thus, to reduce UV-induced ROS generation in the skin, the cosmetics industry has been educating consumers about both the importance of using sunscreen products and their proper use.

Meanwhile, UV absorbers, the active components of sunscreen products, protect the skin by chemically absorbing certain UV wavelengths. In general, since some UV absorbers generate ROS by working as photosensitizers, the Pharmaceutical Affairs Law is concerned about their possible negative influences on skin health ${ }^{10-16)}$. Among UV absorbers, $t$-butyl methoxydibenzoylmethane (BMDM) is widely accepted as an effective UVA absorber and is allowed to be formulated in sunscreen products by the US Food and Drug Administration(FDA), which strictly regulates the use of UV absorbers. Furthermore, the chemical properties of BMDM, which has a broad band spectrum and a high molar absorbance coefficient, apparently provide effective protection against UVA-induced skin damage. However, BMDM is also known for its gradually reduced UVA absorption during prolonged exposure to sunlight. This is termed as "photodegradation," which then allows ROS generation ${ }^{17-32)}$. The use of sunscreen products formulated with BMDM is an ongoing concern since it is possible that consumers are not aware of their own exposure to excessive UVA as a result of BMDM's photodegradation. Accordingly, other UVA absorbers have been developed to overcome that disadvantage of BMDM. However, many of those have failed to achieve the same broad band absorption level as BMDM, which can absorb from $310 \mathrm{~nm}$ to 390 nm.

The purpose of this study was to find appropriate combinations of BMDM and secondary UV absorbers which can prevent skin injury by reducing ROS generation while also controlling UVA absorption.

\section{Experimental}

\subsection{Reagents}

tert-Butyl methoxydibenzoylmethane (BMDM; Parsol1789) was obtained from DSM Nutritional Products (Heerlen, Nederland B.V.). 2-Hydroxy-4-methoxybenzophenone (BP3; Eusolex ${ }^{\circledR} 4360$ ), octyl methoxycinnamate (OMC; Eusolex ${ }^{\circledR} 2292$ ), and octocrylene (OCR; Eusolex ${ }^{\circledR}$
OCR) were purchased from Merck (Darmstadt, Germany).

Glyceryl tri-2-ethylhexanoate(SG-TRIFAT S-308) was obtained from Nikko Chemicals (Tokyo, Japan). Petrolatum was purchased from Koshiro Co. Ltd. (Osaka, Japan). 5,5-Dimethyl-1-pyrroline-N-oxide (DMPO) was purchased from Tokyo Chemical Industry (Tokyo, Japan). Bovine superoxide dismutase (SOD) and Neutral Red (NR) were purchased from Sigma-Aldrich (St. Louis, MO, USA). Dulbecco's modified Eagle medium (DMEM) and Hanks' balanced salt solution with $\mathrm{Ca}^{2+}$ and $\mathrm{Mg}^{2+}$ (HBSS) were obtained from Nissui Pharmacy (Tokyo, Japan) and fetal bovine serum (FBS) was obtained from Invitrogen (Carlsbad, CA, USA).

\subsection{Cell culture}

HaCaT keratinocytes (human immortalized keratinocytes) were cultured in DMEM containing $5 \% \mathrm{FBS}$ at $37^{\circ} \mathrm{C}$ in a humidified atmosphere containing $5 \% \mathrm{CO}_{2}$.

\subsection{Phototoxicity of UV absorbers}

HaCaT keratinocytes were seeded into 96-well plates at a density of $3.5 \times 10^{4}$ cells/well in DMEM containing $5 \%$ FBS. After $24 \mathrm{~h}$, the cells were placed in HBSS and then exposed to UVA at a density of $10 \mathrm{~J} / \mathrm{cm}^{2}$ in the absence or presence of UV absorbers $(0.5 \mu \mathrm{g} / \mathrm{mL})$. The UVA source used was a FL20s BLB lamp (Toshiba, Tokyo, Japan), and the irradiation energy was measured with a UVX-36 Radiometer (Analytik Jena US, CA, USA). After UVA irradiation, the cells were cultured in DMEM containing 5\% FBS for another $24 \mathrm{~h}$, and were then further incubated for $2 \mathrm{~h}$ in DMEM containing $50 \mu \mathrm{g} / \mathrm{mL}$ NR. After washing with PBS, NR was extracted with a methanol, water, and acetic acid solution (in a 50:49:1 ratio) and absorbance was measured at 550 and $650 \mathrm{~nm}$ with a microplate reader. Cell viability was calculated as the percentage of the difference in absorbance at $550 \mathrm{~nm}$ and at $650 \mathrm{~nm}$ compared to the control cells. The phototoxicity index is expressed as the ratio of viability of cells exposed to UVA in the presence of UV absorbers to the viability of cells exposed to UVA in the absence of UV absorbers.

\subsection{Quantification of radicals from UV-irradiated UV ab- sorbers assessed by the ESR (Electron Spin Reso- nance) Spin Trapping method}

Each UV absorber was solubilized at a concentration of $0.01 \%(\mathrm{w} / \mathrm{v})$ in phosphate-buffered saline (PBS) containing $0.1 \%$ DMSO and was mixed with $90 \mathrm{mM}$ DMPO in a 1:1 ratio. The resulting solution was transferred into an ESR quartz flat cell(Flash Point, Tokyo, Japan). The ESR spectrum of UV absorbers irradiated with solar simulated light (UVA + UVB) at an energy density of $96 \mathrm{~mW} / \mathrm{cm}^{2}$ for $5 \mathrm{~min}$ were recorded using a RFR-30 radical analyzer system (JEOL, Tokyo, Japan) with the following conditions: Frequency: $9.43 \mathrm{GHz}$, Power: $4.00 \mathrm{~mW}$, Magnetic field: $336.5 \pm$ 
$7.5 \mathrm{mT}$, Modulation width: $1.25 \mathrm{mT}$, Sweep time: $0.5 \mathrm{~min}$, and Gain: 250. The light source used was a solar simulator (DRC, Osaka, Japan). The UV irradiation time was determined by monitoring the signal intensities derived from the UV absorbers and varying the irradiation, ranging from 30 sec to $10 \mathrm{~min}$, in a stepwise manner under a fixed UV energy of $96 \mathrm{~mW} / \mathrm{cm}^{2}$. Irradiation energy was measured with a UVX-36 Radiometer. The intensity of radicals generated during irradiation is expressed as the parameter "S/ $\mathrm{M}$," which is the ratio of the signal height of radicals generated under UV irradiation and the height of $\mathrm{MnO}$, which is an external standard ${ }^{32)}$. The major peak signal generated by UVA at around $337 \mathrm{mT}$ was used for the calculation of $\mathrm{S} / \mathrm{M}$.

\subsection{Photodegradation of UVA and UVB absorbers}

UV absorbers were dissolved at a concentration of $0.1 \%$ $(\mathrm{w} / \mathrm{w})$ in a mixture of glyceryl tri-2-ethylhexanoate and petrolatum at a ratio of $75: 25$ under heating to $80^{\circ} \mathrm{C}$. The oil gels containing UV absorbers were sandwiched between quartz plates ( $1 \mathrm{~mm}$ thickness) with a spacer that had a thickness of $58 \mu \mathrm{m}$. The UV spectrum of each oil gel was measured with a V-550 UV-visible spectrophotometer (JASCO, Tokyo, Japan) before and after UV irradiation. UV irradiation was carried out with a SPF MASTER ${ }^{\circledR}$ (Shiseido, Tokyo, Japan) at an irradiation density of $1 \mathrm{~mW} / \mathrm{cm}^{2}$ for 60 $\min ^{20)}$. The irradiation energy was measured with a UVX-36 Radiometer. The photodegradation rate was calculated with the following equation using the absorbance at the maximum absorption wavelength of each UV absorber:

Photodegradation rate $(\%)=$

$$
\left(\mathrm{ABS}_{\text {pre }}-\mathrm{ABS}_{\text {post }}\right) \times 100 / \mathrm{ABS}_{\text {pre }}
$$

$\mathrm{ABS}_{\text {pre }}$ : absorbance of pre-irradiation

$\mathrm{ABS}_{\text {post }}$ : absorbance of post-irradiation

\subsection{Statistical analysis}

All data are expressed as means \pm SD. Comparisons between the two groups were performed using the Student's t-test. A $p$-value of less than 0.05 is considered statistically significant.

\section{Results}

3.1 Radical generation and identification of radical species from irradiated UV absorbers

In order to determine the radical generation potential of
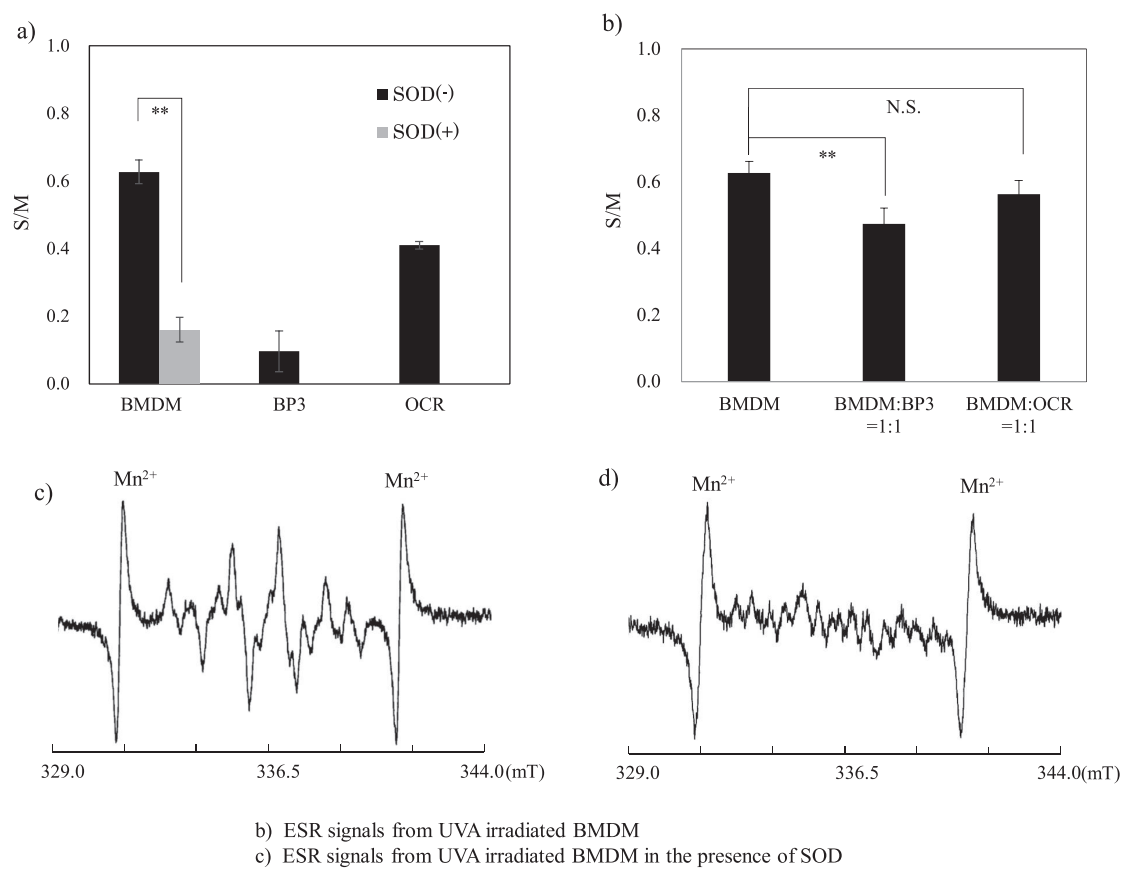

Fig. 1 Radical generation from UV absorbers during UVA irradiation.

UV absorbers were solubilized in phosphate-buffered saline (PBS) containing $0.1 \%$ DMSO at a concentration of $0.01 \%$ and were mixed with DMPO. The resulting solutions were transferred into ESR quartz flat cells and their ESR spectra under irradiating solar simulated light (UVA + UVB) were recorded using a RFR-30 radical analyzer system. a) Radical intensity of UV absorbers irradiated with UV. c) Representative ESR spectrum of BMDM irradiated with UV. d) Representative ESR spectrum of BMDM irradiated with UV in the presence of SOD. b) Radical intensity of BMDM irradiated with UV in the presence of another UV absorber. Data are expressed as means \pm standard deviation in triplicate examinations. Significance; Student t-test *: $p<0.05$, **: $p<0.01$, N.S.: not significant. 
each UV absorber, the radicals generated during UV irradiation were measured using the ESR spin trapping method. Among the UV absorbers examined, BMDM showed a higher radical generation of $0.63 \pm 0.03$ compared with BP3 $(0.10 \pm 0.06)$ and OCR $(0.41 \pm 0.03)$ (Fig. 1a). From their signal patterns, the radicals generated from BMDM were expected to be $\cdot \mathrm{O}_{2}{ }^{-}$. We then examined the effects of SOD (150 units/mL) on radical intensity to identify the radical species involved. The addition of SOD significantly reduced the radicals (Figs. 1c, 1d), indicating that BMDM generated $\cdot \mathrm{O}_{2}{ }^{-}$through a type I photosensitization reaction.

In general, UV absorbers are formulated in products using a mixture of absorbers. In order to reduce the generation of radicals from BMDM during UV irradiation, we added other UV absorbers to BMDM and measured the effects. The addition of BP3 to BMDM significantly reduced radical intensity (Fig. 1b), which suggested that the addition of BP3 into the mixture was an effective solution to inhibit the radical generation from BMDM. In contrast, there was no significant difference when OCT was added to BMDM.

\subsection{Phototoxicity of UV absorbers}

The survival ratios of HaCaT keratinocytes exposed to UVA in the absence or presence of UV absorbers are summarized in Table 1. The concentration of each UV absorber that had no influence on cell viability was used in these experiments. To give us an easy-to-understand measure, we calculated the phototoxicity index as follows: the viability of keratinocytes exposed to UVA in the absence of UV absorbers was divided by the viability of keratinocytes exposed to UVA in the presence of UV absorbers. Although the phototoxicity indexes of BP3 and OCR were significantly lower than that of BMDM, they were not significantly different. Among the UV absorbers examined, BMDM had a significantly higher phototoxicity index due to the large differences in viability in its absence or presence. Interestingly, although the phototoxicity index of BMDM was improved by the addition of BP3 or OCR, the improvement by BP3 was superior to OCR.

\subsection{Photodegradation of UV absorbers}

UV filters formulated in oil gel were placed between quartz plates and were irradiated with simulated solar UV light using a SPF Master. Then, photodegradation behavior was examined for each UV absorber. $\operatorname{BMDM}(41.0 \pm 0.8 \%)$ and $\mathrm{OMC}(44.0 \pm 11.2 \%)$ showed significantly higher photodegradation rates compared with $\mathrm{BP} 3(17.9 \pm 6.2 \%)$ and OCR $(13.4 \pm 4.6 \%$ ) (Fig. 2a), something already well documented in the literature ${ }^{21-27)}$. In order to suppress the photodegradation of BMDM, combinations of BMDM with BP3 or OCR, which showed lower rates of photodegradation, were examined. The combinations of BP3 or OCR significantly suppressed the photodegradation rate of BMDM (Fig. 2b). Next, combinations of BP3 and BMDM were assessed while changing the ratios of BMDM to BP3 to 0.5, 1, 2 and 4 . The degradation rates of BMDM:BP3 were $22.5 \%$ at $0.5: 1,15.3 \%$ at $1: 1,12.8 \%$ at $1: 2$, and $11.6 \%$ at $1: 4$. Adding BP3 strongly decreased the photodegradation of $\operatorname{BMDM}($ Fig. 2b). Those results showed that BP3 suppressed the photodegradation of BMDM. The effect of this suppression was shown even at a ratio of $0.5: 1$. Additional$l y$, it is notable that there was a significant difference between the results of BMDM: BP3 at 0.5:1 and at 1:2, and that the photodegradation rate decreased as the ratio increased.

It has previously been determined that OCR suppresses the photodegradation of UV absorbers ${ }^{26-32)}$. However, the mechanisms of photodegradation of BMDM and of BP3 have not been extensively studied. To characterize the mechanism of action of BP3 in the suppression of BMDM photodegradation, we experimented with UV absorbers sandwiched between quartz plates. BMDM placed on top of BP3 gave the same rate of photodegradation as when BMDM alone was irradiated with UV. These results showed that there was no difference from previous experiments with respect to irradiation energy. In the condition where BMDM was placed under and was irradiated with UV

Table 1 Phototoxicity index.

\begin{tabular}{lcccc}
\hline \multicolumn{1}{c}{ UV absorber } & $\begin{array}{c}\text { Absence of UV } \\
\text { absorber }^{\#}(\%)\end{array}$ & $\begin{array}{c}\text { Presence of } \mathrm{UV} \\
\text { absorber }^{\dagger}(\%)\end{array}$ & significance & phototoxicity index \\
\hline BMDM & $84.8 \pm 6.9$ & $7.6 \pm 1.0$ & $* * *$ & 11.1 \\
BP3 & $88.3 \pm 5.9$ & $72.8 \pm 1.9$ & $* *$ & 1.2 \\
OCR & $88.7 \pm 2.9$ & $79.8 \pm 5.1$ & $*$ & 1.1 \\
BMDM:BP3=1:1 & $73.8 \pm 3.6$ & $27.3 \pm 1.4$ & $* * *$ & 2.7 \\
BMDM:OCR=1:1 & $92.8 \pm 1.8$ & $11.0 \pm 1.5$ & $* * *$ & 8.5 \\
\hline
\end{tabular}

HaCaT keratinocytes were exposed to UVA at a density of $10 \mathrm{~J} / \mathrm{cm}^{2}$ in the presence or absence of a UVA absorber. Cell viability was determined by the incorporation of neutral red. The phototoxicity index is expressed as the ratio of $\#$ and $\dagger$. Data are shown as means \pm standard deviation for quintuplicate examinations. Significance between \# and $\dagger$; Student t-test *: $p<0.05, * *: p<0.01, * * *: p<0.001$ 
(a)

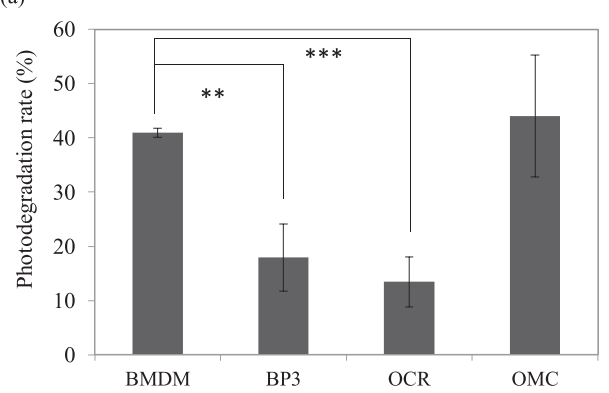

(b)

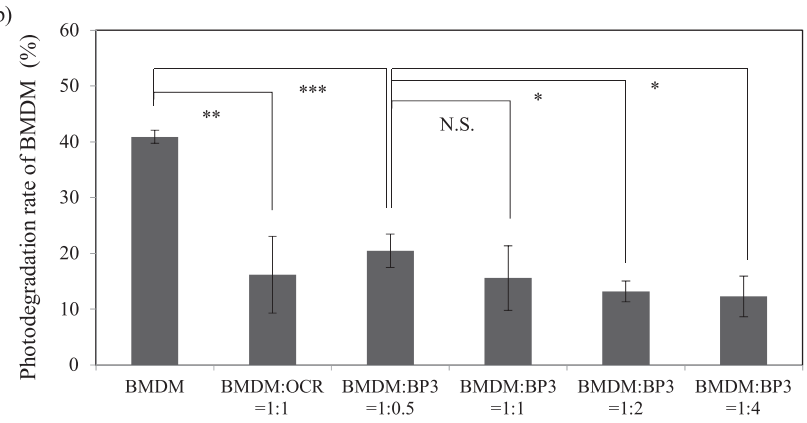

Fig. 2 Photodegradation of UV absorbers during irradiation with UV.

UV absorbers were dissolved in a mixture of glyceryl tri-2-ethylhexanoate and petrolatum, and oil gels containing UV absorbers were sandwiched between quartz plates. The UV spectrum of each oil gel was measured with a V-550 UV-visible spectrophotometer before and after irradiation with simulated solar UV light using a SPF Master. a) Photodegradation rate of each UV absorber, b) Photodegradation rate of BMDM combined with other UV absorbers. The photodegradation rate for (a) is expressed as a percentage of the difference in absorbance at the maximal wavelength for each UV absorber, and for (b) is expressed a percentage of the degradation in absorbance at the maximal wavelength of BMDM. Data are expressed as means \pm standard deviation in triplicate examinations. Significance; Student t-test $* *: p<0.01$, ***: $p<$ 0.001, N.S.: not significant.

through BP3, which was on top, the photodegradation showed a similar rate as BMDM alone, but in contrast, the BMDM mixed with BP3 had a significantly lower photodegradation rate(Fig. 3 ).

\section{Discussion}

UVA absorbers are immensely important as UVA is known to accelerate skin aging, which is characterized by the formation of wrinkles and sagging skin. As BMDM is considered to be a highly effective UVA absorber, its stabil- ity and safety must be closely scrutinized. Indeed, BMDM is widely used in sunscreen products. However, it has the disadvantage of gradually losing its UV absorbance capacity during UV exposure. Previous studies have demonstrated that this loss of UV absorbance capacity, called photodegradation, is related to cis-trans isomerization, the quenching of the triplet diketo form of BMDM, and also to photolysis by photomultiplication resulting from energetic exposure $^{25-31)}$. Thus, although studies on the photodegradation of UV absorbers have been actively conducted, little attention has been paid to the generation of ROS from UV absorbers during UV exposure and the resulting risk of skin damage. ROS is a major factor that causes UV-accelerated skin aging. A recent study using in vivo confocal Raman spectroscopy has demonstrated that UV absorbers easily penetrate into the stratum corneum ${ }^{34-36)}$. The sum of these results has identified that radical generation in the stratum corneum by UV absorbers poses a potential risk not only in enhancing skin aging but also in causing phototoxicity or photoallergy.

This study was conducted to find a suitable combination of BMDM and secondary UV absorbers to prevent skin damage by reducing the generation of ROS while also controlling UVA absorption during UV exposure. The ESR spin trapping study of the absorbers during UVA irradiation showed that BMDM generated radicals at higher intensities compared with the other UV absorbers (Fig. 1a). Since the radical species were presumed to be superoxide anion radicals $\left(\cdot \mathrm{O}_{2}{ }^{-}\right)$from their signal patterns, the effects of SOD on radical intensity were examined. The radical generated was identified as $\cdot \mathrm{O}_{2}{ }^{-}$since SOD dramatically reduced the signal intensity. In general, $\cdot \mathrm{O}_{2}{ }^{-}$is spontaneously converted to $\mathrm{H}_{2} \mathrm{O}_{2}$ and finally to $\cdot \mathrm{OH}$ in the presence of $\mathrm{Fe}^{2+}$ or $\mathrm{Cu}^{+}$. $\cdot \mathrm{OH}$ damages cells at a high frequency. Next, we examined the cell damage caused by UV absorbers during UVA irradiation. HaCaT keratinocytes exposed to UVA in the presence of BMDM showed a significantly lower cell viability compared with the other UV absorbers. Those results indicated that $\cdot \mathrm{O}_{2}{ }^{-}$generated from BMDM during UVA irradiation is highly cytotoxic.

Interestingly, $\cdot \mathrm{O}_{2}{ }^{-}$generation from UVA-irradiated BMDM was suppressed by the addition of BP3 or OCR (Fig. 1a). Meanwhile, BP3 restored the cell viability reduced by BMDM during UVA exposure (Table 1). Collating these results suggested that BMDM can damage the skin, inducing phototoxicity or photoallergy by $\cdot \mathrm{O}_{2}{ }^{-}$generation. Combining BMDM with BP3 seemed to reduce this risk. Regarding the photodegradation of UV absorbers, BP3 also suppressed BMDM's photodegradation rate. As such, BP3 combined with BMDM seems to be a safer and more effective sunscreen to prevent UVA damage to the skin, even compared to OCR, which is generally known as a BMDM stabilizer.

The mechanism by which BP3 suppresses radical gener- 
K. Yamaguchi, R. Ohtsuka, K. Kaneko et al.

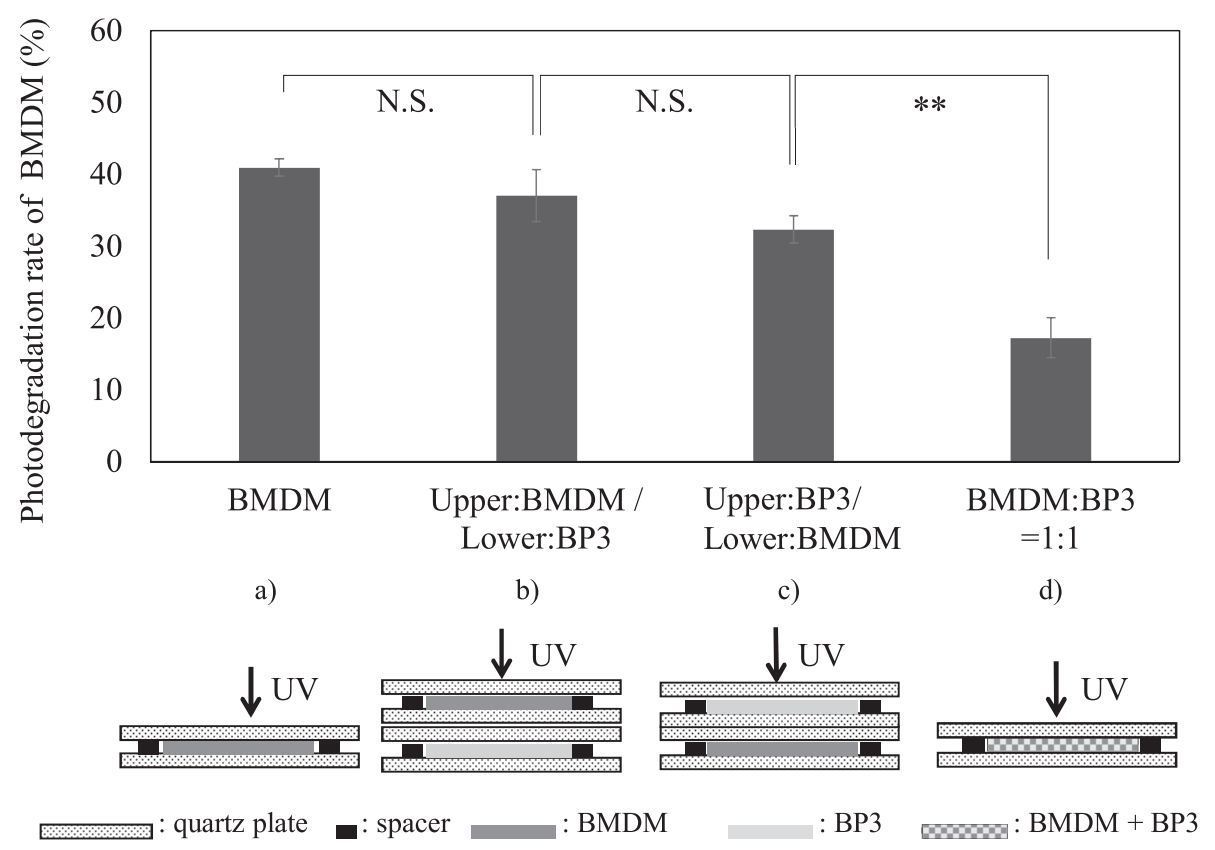

Fig. 3 Effects of OCR and BP3 on the photodegradation of BMDM.

BMDM with or without BP3 was dissolved in a mixture of glyceryl tri-2-ethylhexanoate and petrolatum and was sandwiched between quartz plates with a spacer. Each sandwiched plate was stacked as follows: upper side/bottom side, a) BMDM, b) BMDM/BP3, c) BP3/BMDM, d) quartz plate/BMDM + BP3. The plates were irradiated with UV from the upper side. The UV spectrum of each sandwiched plate was measured with a V-550 UV-visible spectrophotometer before and after irradiation with simulated solar UV light using a SPF Master. The photodegradation rate(\%)is expressed as a percentage of the difference in absorbance at the maximal wavelength of each UV absorber. Data are expressed as means \pm standard deviation in triplicate examinations. Significance; Student t-test $*: p<0.05, * *: p<$ $0.01, * * *: p<0.001$, N.S.: not significant.

ation and the photodegradation of BMDM was examined. First, we hypothesized that the region of wavelength absorbed by BP3 is responsible for the photodegradation of BMDM. In order to investigate the mechanism of action of $\mathrm{BP} 3$, we examined the filtering effect of BP3 against the absorption spectrum of BMDM using photodegradation. UV light extracted with the BP3 filter caused a similar degree of photodegradation to BMDM exposed to non-filtered UV light, which disproved that the wavelength absorbed by BP3 is responsible for the generation of $\cdot \mathrm{O}_{2}{ }^{-}$and the photodegradation of BMDM. The next hypothesis speculated that a chemical interaction of BMDM and BP3 was responsible. In order to test that hypothesis, the behavior of BMDM photodegradation in the presence of BP3 was examined. BP3 showed a significant suppression of BMDM photodegradation when mixed together (Fig. 3). In fact, OCR has been known to be a stabilizer of BMDM due to its function as a receptor of UV-excitation energies in $\mathrm{BMDM}^{21-25}$. Furthermore, it has been reported that UVB absorbers prevent the breakdown of BMDM by triplet-triplet energy transfers using photoinduced triplet-excited states $^{26-32)}$. The results of this study suggest that BP3 works as a receptor of the excited energy of BMDM exposed to UV.

With respect to the relationship between superoxide generation and phototoxicity, although the combination of BP3 to BMDM reduced radical generation, the degree of the reduction was not as high as that of BMDM in the presence of SOD or that of BP3 alone. The reduction of BMDMderived phototoxicity in the presence of BP3 might be explained by the reduction of radical generation being lower than the threshold concentration. As it is known that cell death has a threshold concentration of hydrogen peroxide, we inferred that there is a threshold concentration of ROS necessary to cytotoxicity ${ }^{33)}$. In addition, we saw differences between the reduction in radical generation from UV absorbers under UVA exposure and the suppression of phototoxicity. Although it is recognized that the radicals derived from UVA-exposed UV absorbers are responsible for the cytotoxicity, it is hard to rule out the possibility that intermediates of the UV absorbers generated under UVA exposure cause cytotoxicity. The difference between them might appear from differences in the toxicity of their intermediates.

Considering the results of $\mathrm{BP} 3$ and OCR on the reduction of radical generation and the suppression of photodegradation during UV exposure, the effects were shown not to be parallel between those two processes. In general, photo-induced radical generation progresses by photo-sen- 
sitization. In the case of BMDM, the absorbed UVA energy generates superoxide anion radicals by electron transfers through Type I photosensitization reactions. On the other hand, the photodegradation of BMDM has been demonstrated to reduce the absorbance at certain UVA regions due to the photo-isomerization of the enol form from trans to cis. Reductions of radical generation may be dependent on the ability to quench excited energies, and the suppression of photodegradation may be realized by restricting conformational changes of the enol form due to solvation by OCR. The differences between BP3 and OCR in reducing radical generation and suppressing photodegradation probably depend on their energy-quenching properties and the restriction of conformational changes during UVA exposure. However, since we don't have any results to discuss these differences, it will require further study.

In order to identify the action of BP3 on BMDM, further studies are required. We plan to continue exploring these issues and will report our further findings in the future.

\section{Conclusion}

In this study, we demonstrated that BMDM, the most widely-used UVA absorber in cosmetic sunscreen products, photodegrades and becomes cytotoxic, which was caused by the generation of $\cdot \mathrm{O}_{2}{ }^{-}$during UV exposure. In fact, it has been previously reported that BMDM is responsible for the induction of phototoxicity and photoallergy ${ }^{34-39)}$. In order to propose a solution to formulate BMDM in sunscreen products more safely and stably, we found that the combination of BMDM and BP3 was effective. Furthermore, it is expected that this proposed combination will delay skin aging as it effectively and safely blocks UV radiation.

\section{References}

1) Gunn, D.A.; Rexbye, H.; Griffiths, C.E.M.; Murray, P.G.; Fereday, A,; Catt, S.D. et al. Why some women look young for their age. PLoS One 4, e8021 (2009).

2) Kligman, L.H. Photoaging: Manifestations, prevention, and treatment. Clin. Geriatr. Med. 5, 235-251 (1989).

3) Baier, J.; Maisch, T.; Maier, M.; Engel, E.; Landthaler, M.; Baumler, W. Singlet oxygen generation by UVA light exposure of endogenous photosensitizers. Biophys. J. 91, 1452-1459(2006).

4) Masaki, H.; Izutsu, Y.; Yahagi, S.; Okano, Y. Reactive oxygen species in HaCaT keratinocytes after UVB irradiation are triggered by intracellular $\mathrm{Ca}^{2+}$ levels. J. Invest. Dermatol. Symp. Proc. 14, 50-52(2009).

5) Li, Y.; Xia, W.; Liu, Y.; Remmer, H.A.; Voorhees, J.; Fisher, G.J. Solar ultraviolet irradiation induces deco- rin degradation in human skin likely via neutrophil elastase. PLoS One 8, e72563 (2013).

6) Sakurai, H.; Yasui, H.; Yamada, Y.; Nishimura, H.; Shigemoto, M. Detection of reactive oxygen species in the skin of live mice and rats exposed to UVA light: A research review on chemiluminescence and trials for UVA protection. Photochem. Photobiol. Sci. 4, 715720 (2005).

7) Chiba, K.; Kawakami, K.; Sone, T.; Onoue, M. Characteristics of skin wrinkling and dermal changes induced by repeated application of squalene monohydroperoxide to hairless mouse skin. Appl. Skin Physiol. 16, 242-251 (2003).

8) Cortat, B.; Garcia, C.C.; Quinet, A.; Schuch, A.P.; de Lima-Bessa, K.M.; Menck, C.F. The relative roles of DNA damage induced by UVA irradiation in human cells. Photochem. Photobiol. Sci. 12, 1483-95 (2013).

9) Wan, Y.; Belt, A.; Wang, Z.; Voorhees, J.; Fisher, G.J. Transmodulation of epidermal growth factor receptor mediates IL-1 beta-induced MMP-1 expression in cultured human keratinocytes. Int. J. Mol. Med. 7, 329334 (2001).

10) Hanson, K.M.; Gratton, E.; Bardeen, C.J. Sunscreen enhancement of UV-induced reactive oxygen species in the skin. Free Radic. Biol. Med. 41, 1205-1212 (2006).

11) Hayden, C.G.; Cross, S.E.; Anderson, C.; Saunders, N.A.; Roberts, M.S. Sunscreen penetration of human skin and related keratinocyte toxicity after topical application. Skin Pharmacol. Physiol. 18, 170-174 (2005).

12) Karlsson, I.; Hillerstrom, L.; Stenfeldt, A.L.; Martensson, J.; Borje, A. Photocatalytic allergy to dibenzoylmethane is caused by arylglyoxals formed upon photolysis. Chem. Res. Toxicol. 22, 1881-1892(2009).

13) Asai, M.; Kawada, A.; Aragane, Y.; Yudate, T.; Isogai, R.; Tezuka, T. Contact dermatitis due to octyl methoxycinna-mate and butyl methoxydibenzoylmethane with photo-aggravation. Environ. Dermatol. 8, 28-30 (2001).

14) De Groot, A.C.; Weyland, J.W. Contact allergy to butyl methoxydibenzoylmethane. Contact Dermatitis. 16, 278 (1987).

15) Schauder, S.; Ippen, H. Photoallergic and allergic contact dermatitis from dibenzoylmethanes. Photodermatol. 3, 140-147 (1986).

16) Onoue, S.; Tsuda, Y. Analytical studies on the prediction of photosensitive/phototoxic potential of pharmaceutical substances. Pharm. Res. 23, 156-164(2006).

17) Nash, J.F.; Tanner, P.R. Relevance of UV filter/sunscreen product photostability to human safety. Photodermatol. Photoimmunol. Photomed. 30, 88-95 (2014).

18) Sayre, R.M.; Dowdy, J.C.; Gerwig, A.J.; Shields, W.J.; 


\section{K. Yamaguchi, R. Ohtsuka, K. Kaneko et al.}

Lloyd, R.V. Unexpected photolysis of the sunscreen octinoxate in the presence of the sunscreen avobenzone. Photochem. Photobiol. 81, 452-456 (2005).

19) Gonzalez, H.; Tarras-Wahlberg, N.; Stromdahl, B.; Juzeniene, A.; Moan, J.; Larko, O.; Rosen, A.; Wennberg, A. Photostability of commercial sunscreens upon sun exposure and irradiation by ultraviolet lamps. BMC Dermatol. 7, 1(2007).

20) Miura, Y.; Takiguchi, Y.; Shirao, M.; Takata, S.; Yanagida, T.; Fukui, H.; Naganuma, M.; Hatao, M. Algorithm for in vitro sun protection factor based on transmission spectrum measurement with concomitant evaluation of photostability. Photochem. Photobiol. 84, 1569-1575 (2008).

21) Mendrok-Edinger, C.; Smith, K.; Janssen, A.; Vollhardt, J. The quest for avobenzone stabilizers and sunscreen photostability. Cosmet. Toiletries 124, 47-54(2009).

22) Kockler, J.; Oelgemöller, M.; Robertson, S.; Glass, B.D. Photostability of sunscreens. J. Photochem. Photobiol. C 13, 91-110 (2012).

23) Durango, S.; Castañeda, S.; Vallejo, J.; Gallardo, C. Solvent effect on photostability of butyl methoxy di benzoyl methane formulated in solution and emulsion. Int. J. Pharm. Sci. 7 (9), 181-186(2015).

24) Maier, H.; Schauberger, G.; Brunnhofer, K.; Honigsmann, H. Change of ultraviolet absorbance of sunscreens by exposure to solar-simulated radiation. $J$. Invest. Dermatol. 117, 256-262(2001).

25) Tarras-Wahlberg, N.; Stenhagen, G.; Larko, O.; Rosen, A.; Wennberg, A.M.; Wennerstrom, O. Changes in ultraviolet absorption of sunscreens after ultraviolet irradiation. J. Invest. Dermatol. 113, 547-553 (1999).

26) Paris, C.; Lhiaubet-Vallet, V.; Jimenez, O.; Trullas, C.; Miranda, M.A. A blocked diketo form of avobenzone: Photostability, photosensitizing properties and triplet quenching by a triazine-derived UVB-filter. Photochem. Photobiol. 85, 178-184(2009).

27) Lhiaubet-Vallet, V.; Marin, M.; Jimenez, O.; Gorchs, O.; Trullas, C.; Miranda, M.A. Filter-filter interactions. Photostabilization, triplet quenching and reactivity with singlet oxygen. Photochem. Photobiol. Sci. 9, 552-558 (2010).

28) Marin, M.; Lhiaubet-Vallet, V.; Paris, C.; Minoru, Y.; Miranda, M.A. Photochemical and photophysical properties of dibenzoylmethane derivatives within protein. Photochem. Photobiol. Sci. 10, 1474-1479(2011).
29) Yamaji, M.; Kida, M. Photothermal tautomerization of a UV sunscreen (4-tert-butyl-4'-methoxydibenzoylmethane) in acetonitrile studied by steady-state and laser flash photolysis. J. Phys. Chem. A 117, 1946-1951 (2013).

30) Oguchi-Fujiyama, N.; Miyazawa, K.; Kikuchib, A.; Yagi, M. Photophysical properties of dioctyl 4-methoxybenzylidenemalonate: UV-B absorber. Photochem. Photobiol. Sci. 11, 1528-1535(2012).

31) Kikuchi, A.; Oguchi-Fujiyama, N.; Miyazawa, K.; Yagi, M. Triplet-triplet energy transfer from a UV-A absorber butylmethoxydibenzoylmethane to UV-B absorbers. Photochem. Photobiol. 90, 511-516(2014).

32) Noda, Y.; Anzai, K.; Mori, A.; Kohno, M.; Shinmei, M.; Packer, L. Hydroxyl and superoxide anion radical scavenging activities of natural source antioxidants using the computerized JES-FR30 ESR spectrometer system. Biochem. Mol. Biol. Int. 42, 35-44(1997).

33) Burdon, R.H. Superoxide and hydrogen peroxide in relation to mammalian cell proliferation. Free Radic. Biol. Med. 18, 775-794(1995).

34) Simeoni, S.; Scalia, S.; Benson, H.A.E. Influence of cyclodextrins on in vitro human skin absorption of the sunscreen, butyl-methoxydibenzoylmethane. Int. J. Pharmaceut. 280, 163-171 (2004).

35) Iannuccelli, V.; Coppi, G.; Sergi, S.; Mezzena, M.; Scalia, S. In vivo and in vitro skin permeation of butyl methoxydibenzoylmethane from lipospheres. Skin Pharmacol. Physiol. 21, 30-38(2008).

36) Lasarow, R.M.; Isseroff, R.R.; Gomez, E.C. Quantitative in vitro assessment of phototoxicity by a fibroblastneutral red assay. J. Invest. Dermatol. 98, 725-729 (1992).

37) Onoue, S.; Igarashi, N.; Yamauchi, Y.; Kojima, T.; Murase, N.; Zhou, Y.; Yamada, S.; Tsuda, Y. In vitro phototoxic potential and photochemical properties of imidazopyridine derivative: A novel 5-HT4 partial agonist. J. Pharm. Sci. 97, 4307-4318(2008).

38) Schauder, S.; Ippen, H. Photoallergic and allergic contact eczema caused by dibenzoylmethane compounds and other sunscreening agents. Hautarzt 39, 435-440 (1988).

39) Tippavajhala, V.K.; de Oliveira Mendes, T.; Martin, A.A. In vivo human skin penetration study of sunscreens by confocal Raman spectroscopy. AAPS PharmSciTech. 19, 753-760 (2017). 